\title{
Was ist bei der Bildung von Qualitätsindikatoren aus Routinedaten zu beachten?
}

\section{Ausgangspunkt \\ $\checkmark$}

Im Gegensatz zum Ausland gibt es in Deutschland bislang kaum Erfahrungen mit der Messung und Bewertung der Qualität der medizinischen Leistungserbringung anhand von Routinedaten der gesetzlichen Krankenkassen. Vielmehr stützten sich Verfahren der externen Qualitätssicherung bislang auf aufwändige spezielle Datenerhebungen. Im QSR-Projekt wurde nun erstmals ein Konzept für eine Qualitätssicherung mit Routinedaten entwickelt [1].

\section{Datengrundlage \\ $\nabla$}

Der QSR-Ansatz basiert auf den Routinedaten der gesetzlichen Krankenversicherung. Diese haben sich in den letzten Jahren zunehmend als valide Datenquelle für die Versorgungsforschung etabliert [2]. Ihre Vorteile liegen u.a. in der Vollständigkeit, Aktualität, kostengünstigen Verfügbarkeit und ihrem eindeutigen Versicherungsbezug. Auch die Datenqualität ist im allgemeinen als gut zu bezeichnen, muss jedoch vor dem Hintergrund der konkreten Fragestellung explizit geprüft werden. Das gilt auch für eine potenzielle (Sekundär-) Nutzung zur Ergebnismessung und -bewertung.

\section{Methodische Aspekte der Nutzung von Routinedaten \\ $\checkmark$}

Ein routinedatengestütztes Verfahren zur Qualitätsdarstellung (des stationären Bereichs) muss die klinische Situation in den Krankenhäusern möglichst verzerrungsfrei abbilden und zusätzlich einen validen Vergleich der Leistungserbringer untereinander erlauben. $\mathrm{Zu}$ diesen beiden Punkten gibt es gesonderte Tagungsbeiträge zum Traceransatz und zur Risikoadjustierung innerhalb des QSR-Ansatzes. Auf weitere methodische Aspekte wird in diesem Beitrag eingegangen. So können GKV-Routinedaten patientenbezogen mittel- und langfristige Ergebnisse der Behandlung abbilden, wenn der Zugriff auf Versichertenstammdaten gegeben ist. Es muss dabei geprüft werden, inwieweit mit zunehmender Nachbeobachtungszeit beobachtbare Ergebnisindikatoren noch Ausdruck der primären Behandlung sind. Bei der tracerspezifischen Definition von Qualitätsindikatoren ist weiterhin festzulegen, ob Einzelindikatoren oder ein zusammengesetzter Qualitätsindex Basis der Qualitätsbewertung sein sollen. Noch nicht befriedigend gelöst ist weiterhin die sektorübergreifende Abbildung und Bewertung von Behandlungsverläufen, vor allem in Bezug auf die Validität der Diagnosen im ambulanten Sektor (vgl. den Beitrag von Schubert). Weitere methodische Fragen betreffen die Minimierung des lost-of-follow-up (etwa durch Versicherungswechsel), den Umgang mit kleinen Fallzahlen oder die kassenübergreifende Qualitätsmessung. (Bislang basiert der QSR-Ansatz allein auf AOK-Daten.)

\section{Schlussfolgerung}

Die Qualitätsmessung und -bewertung anhand von GKV-Routinedaten ist nach den Erfahrungen des QSR-Projekts grundsätzlich möglich und eröffnet gegenüber der externen Qualitätssicherung vielfältige neue Einblicke in das Leistungsgeschehen [3]. Es wird gemeinsame Aufgabe von Leistungserbringern, Kostenträgern und Wissenschaft sein, für die offenen methodischen Fragen wissenschaftlich belastbare wie praktisch umsetzbare Lösungen zu finden.

Autorenerklärung: Der Autor erklärt, dass keine relevanten finanziellen Verbindungen in Bezug auf dieses Manuskript bestehen.

\section{Literatur}

1 AOK-Bundesverband, Forschungs- und Entwicklungsinstitut für das Sozial- und Gesundheitswesen Sachsen-Anhalt (FEISA), HELIOS Kliniken, Wissenschaftliches Institut der AOK (WIdO) (Hrsg.). Qualitätssicherung der stationären Versorgung mit Routinedaten (QSR). Abschlussbericht. Bonn: 2007

2 Swart E, Ihle P (Hrsg.). Routinedaten im Gesundheitswesen. Handbuch Sekundärdatenanalyse: Grundlagen, Methoden und Perspektiven. Bern: Hans Huber, 2005

3 Heller G. Zur Messung und Darstellung von medizinischer Ergebnisqualität mit administrativen Routinedaten in Deutschland. Bundesgesundheitsblatt - Gesundheitsforschung - Gesundheitsschutz, 2008; im Druck

\section{E. Swart}

Qualitätsmanagement

Schlüsselwörter

Routinedaten

$\checkmark$ Qualitätsmessung

Qualitätsindikatoren

Key words

administrative data

quality measurement

quality indicators

Institut

Institut für Sozialmedizin und Gesundheitsökonomie, Medizinische Fakultät der Otto-von-Guericke-Universität Magdeburg

Bibliografie

DOI 10.1055/s-0028-1085597

Dtsch Med Wochenschr 2008;

133: S153 - (c) Georg Thieme

Verlag KG Stuttgart - New York . ISSN 0012-0472

Korrespondenz

Dr. Enno Swart

Institut für Sozialmedizin und

Gesundheitsökonomie

Medizinische Fakultät der

Otto-von-Guericke-Universität

Magdeburg

Leipziger Str. 44

39120 Magdeburg

Tel. +493916724306

eMail

enno.swart@med.ovgu.de 